4. Zusammenfassung: Die vorstehenden Ausführungen haben dargetan, daß das beschriebene Instrument für den angegebenen Spannungsbereich von I bis 5 Volt und Frequenzbereich von 40 bis 60 sowie die üblichen Schwankungen der Außentemperatur genügend genau und mit einem Stromverbrauch von höchstens $0,7 \mathrm{~mA}$. Für höhere Spannungen läßt es sich durch Anbringen von Anzapfungen an der Oberspannungswicklung oder Verwendung statischer Instrumente für hohe Spannungen brauchbar machen, für nicht zu erheblich kleinere durch Anzapfungen an der Unterspannung. Für Frequenzen unter 40 würde man den zu großen nacheilenden Strom durch Anschluß eines Kondensators kompensieren können. Von der Kurvenform ist die Anzeige des Instruments praktisch unabhängig. Der Wandler überträgt die Kurve naturgetreu, zumal bei so kleinen Sättigungen. Einige Stichproben mit verzerrten Wellen bestätigten die Überlegung.

Das Äußere der Meßeinrichtung ist aus Abb. I6 ersichtlich; ein gewöhnlicher. Instrumentenbock trägt oben das Elektrometer und unten den Wandler. Mittels Traggriffen läßt er sich leicht an die gewünschte Meßstelle schaffen.

Zur Lieferung des Instrumentariums sind die Firmen Siemens \& Halske sowie Hartmann \& Braun bereit.

\title{
Die Maßsysteme, die die elektromagnetischen Größen an die mechanischen anschließen.
}

Von

Walter Dällenbach, Zürich.

Es gibt schon sehr viele Arbeiten über diesen Gegenstand. Fast allen ist eigen, daß sie in voller Allgemeinheit, die überhaupt möglichen Maßsysteme entwickeln und in ausführlichen Tabellen Größe und Dimension der verschiedenen Einheiten und ihrer Bezi ehungen untereinander zusammenstellen.

Mir scheint, dabei kam das physikalische Verstehen etwas zu kurz. Ich habe mich demgegenüber bemüht, durch Vermeiden jeglichen Schematismus, die Aufmerksamkeit des Lesers gerade auf die paar physikalischen Schwierigkeiten zu lenken, die immer wieder aufzutauchen pflegen, da bislang eine einfache zusammenfassende Klärung in der Literatur fehlte.

Die Voraussetzung einer Erörterung über Maßsysteme ist die Kenntnis, der die betreffenden physikalischen Größen verknüpfenden Theorie.

I. Die Maxwellsche Theorie des elektromagnetischen Feldes. Es bedeuten die Vektoren $e$ und $\mathfrak{h}$ die elektrische bzw. magnetische Feldstärke, $\mathfrak{d}$ die dielektrische Verschiebung und $\mathfrak{b}$ die magnetische Induktion.

$\mathfrak{e}, \mathfrak{h}, \mathfrak{b}, \mathfrak{b}$ sind verknüpft mit der wahren elektrischen Ladungsdichte $\varrho$ und der Leitungsstromdichte $\mathfrak{i}$ : durch das als die Maxwellschen Feldgesetze bezeichnete System von partiellen Differentialgleichungen:

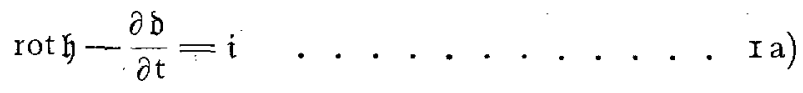

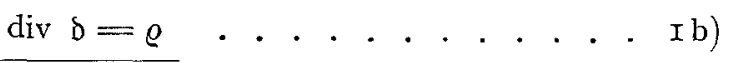

$$
\begin{aligned}
& \text { rote } \left.+\frac{\delta \mathfrak{b}}{\partial t}=0 \quad \ldots . . . . . . . I C\right) \\
& \operatorname{div} \mathfrak{b}=0 \quad \ldots . . . . . . . . I \mathrm{I})
\end{aligned}
$$


Diese Gleichungen I) wie auch die in der Folge auftretenden Gleichungen 3) und 4) sind, solange wir keine Maßeinheiten haben, nur nach der durch sie zwischen den Zustandsgrößen gesetzten allgemein-funktionellen Abhängigkeit von Bedeutung. Wir schreiben daher alle noch unserer Willkür unterworfenen konstanten Multiplikatoren in ihnen vorläufig gleich eins und notieren die Beziehungen in der denkbar einfachsten Gestalt. Erst im Abschnitt 2 werden wir darauf zurückkommen und in anderer Weise verfügen.

Um an die anschauliche Bedeutung der Gleichungen I) zu erinnern, folge jetzt die sogenannte Integralform, und schlieBlich spreche ich ihren Inhalt in W'orten aus:

$$
\begin{aligned}
& \left.\mathrm{H}=\int(\mathfrak{h}, \mathrm{d} \mathrm{l})=\frac{\partial}{\partial \mathrm{t}}\left\{\int(\mathrm{b}, \mathrm{do})\right\}+\int(\mathfrak{i}, \mathrm{do})=\frac{\partial \Psi}{\partial \mathrm{t}}+\mathrm{i} \ldots \ldots .2 \mathrm{a}\right) \\
& \left.\Psi=\int(\mathrm{b}, \mathrm{do})=\int \varrho \mathrm{dt}=\mathrm{e} \quad . \quad . \quad . \quad . \quad . \quad . \quad 2 \mathrm{~b}\right) \\
& \mathrm{E}=\int(\mathfrak{e}, \mathrm{d} \mathrm{l})=-\frac{\partial}{\partial \mathrm{t}}\left\{\int(\mathfrak{b}, \mathrm{do})\right\}=-\frac{\partial \Phi}{\partial \mathrm{t}} \text {........ . . } 2 \mathrm{c} \text { ) } \\
& \left.\Phi=\int(\mathfrak{b}, \mathrm{do})=0 \quad . \quad . \quad . \quad . \quad . \quad . \quad . \quad .2 \mathrm{~d}\right)
\end{aligned}
$$

a) Die magnetische Spannung $\mathrm{H}=\int(\mathfrak{h}, \mathrm{dl})$ längs einer geschlossenen Raumkurve ist gleich dem Verschiebungsstrom $\frac{\partial \Psi}{\partial \mathrm{t}}$ plus dem Leitungsstrom $\mathrm{i}$ durch irgendeine in die Raumkurve eingespannte Fläche.

b) Der Verschiebungsfluß $\Psi$ durch irgendeine geschlossene Oberfläche ist gleich der in ihr eingeschlossenen Elektrizitätsmenge e.

c) Die elektrische Spannung $\mathrm{E}=\int(\mathrm{e}, \mathrm{d} l)$ längs einer geschlossenen Raumkurve ist gleich der Abnahme des Induktionsflusses -- $\frac{\partial \Phi}{\partial \mathrm{t}}$ durch irgendeine in die Raumkurve eingespannte Fläche. Das negative Vorzeichen gegenüber $\frac{\partial \Psi}{\partial \mathrm{t}}$ in a) ist Ausdruck der
Lenzschen Regel.

d) Der Induktionsfluß $\Phi$ durch irgendeine geschlossene Oberfläche ist null. Es. gibt keinen wahren Magnetismus.

Sind in einem bestimmten Zeitmomente die räumliche Verteilung von Strom- und Ladungsdichte $\mathfrak{i}$ und $\varrho$ gegeben, so sollte es möglich sein, aus den Feldgesetzen I) die Vektorfelder $\mathfrak{e} ; \mathfrak{h}, \mathfrak{b}, \mathfrak{b}$ durch Integration $z \mathfrak{u}$ berechnen. Das ist aber nicht der Fall; denn die 4 Vektoren haben total I2 Komponenten, und die 8 Komponentengleichungen I) genügen nicht zur Bestimmung von $\mathrm{x} 2$ unabhängigen Raumfunktionen.

Es bestehen aber noch weitere Beziehungen, die sogenannten Materialgleichungen. Sie ordnen an jeder Stelle des Raumes einem zeitlichen Verlaufe der Feldstärken einen ganz bestimmten Verlauf der dielektrischen Verschiebung bzw. der magnetischen Induktion zu

$$
\left.\begin{array}{l}
\mathfrak{b}=\mathfrak{b}(\mathfrak{e}) \\
\mathfrak{b}=\mathfrak{b}(\mathfrak{h})
\end{array}\right\}
$$

Im scharfen Gegensatz zu den sehr allgemein gültigen Feldgesetzen enthalten diese Gleichungen die ganze Tücke und Mannigfaltigkeit der Materie. Für den leeren Raum, den Äther, sowie für viele homogene und isotrope Körper gilt

$$
\left.\begin{array}{l}
\mathfrak{b}=\varepsilon \mathfrak{e} \\
\mathfrak{b}=\mu \mathfrak{h}
\end{array}\right\}
$$

wo $\varepsilon$ und $\mu$ für die Substanz charakteristische Konstanten bedeuten. Bei inhomogenen Körpern variieren $\varepsilon$, und $\mu$ von Ort zu Ort. Für Ferromagnetica hängt $\mu$ von der Feldstärke selbst ab und wird bei Anwesenheit von Hysterese sogar bedingt durch die Vorgeschichte. In Kristallen gilt für jede Komponente von $\mathfrak{b}$ bzw. $\mathfrak{b}$ eine be- 
sondere Abhängigkeit, will sagen, $\mathfrak{d}$ und $\mathfrak{e} b z w$. $\mathfrak{b}$ und $\mathfrak{h}$ fallen im allgemeinen nicht einmal mehr ihrer Richtung nach zusammen. Endlich gehen in die Materialgleichungen oft auch die zeitlichen Ableitungen der Feldstärken (Dispersion), die Temperatur (Elektro- und magnetokalor. Effekte), der elastische Zustand (Elektrostriktion) usw. als unabhängige Veränderliche ein. In ihnen steckt wirklich die vorläufig ganz unfaßbare Kompliziertheit der Materie, von der gerade die Feldgesetze unberührt bleiben. Wie wichtig dieser Umstand für die Elektrotechnik ist, weiß jeder, der sich beispielsweise der durchgehenden Bedeutung des Induktionsgesetzes bewußt ist.

Für unsere Zwecke beschränken wir uns in der Folge auf homogene, isotrope Körper mit konstantem $\varepsilon$ und $\mu$ gemäß Gleichungen 3 ).

Die 8 Komponentengleichungen I) haben damit einen Zuwachs von weiteren 6 erfahren. Die zu berechnenden I2 Komponenten von $\mathfrak{e}, \mathfrak{h}, \mathfrak{b}, \mathfrak{b}$ haben I4 Gleichungen zu genügen, sind also überbestimmt. Doch nur scheinbar, denn die beiden Divergenzgleichungen I b) und Id) sind nicht unabhängig von Ia) bzw. Ic), wie man erkennt durch Ausüben der Operation div auf die letzteren.

I 2 Gleichungen für I 2 Unbekannte: die Vektorfelder $e, \mathfrak{h}, \delta, \mathfrak{b}$ sind zu berechnen und seien berechnet.

Das Feld übt mechanische Kräfte auf Strom und Ladungen aus. Was uns in teressiert ist die Kraftdichte $k$, das ist die Kraft auf die Volumeneinheit an jedem Ort. An Stelle einer Ableitung geben wir hier das Resultat

$$
\mathrm{k}=\varrho \rho+[\mathfrak{i}, \mathfrak{b}] \text {. . . . . . . . . 4) }
$$

Außer diesen Kräften auf Ladungs- und Stromdichte erfährt die Materie noch Kräfte infolge ihres Polarisationszustandes, die wir aber vorläufig weglassen.

Diese dynamische Gleichung 4) vermittelt den Anschluß an die Mechanik; denn der Wert von $\mathrm{k}$ in die mechanischen Gleichungen eingesetzt, ergibt die Bewegungen der vorhandenen Körper und damit Strom- und Ladungsverteilung im nächsten Zeitmoment, auf Grund derer das Feld von neuem zu berechnen ist, und so fort.

Die im Innern von Leitern frei bewegliche Elektrizität folgt den Änderungen des elektrischen Feldes fast augenblicklich und nimmt wie ganz feiner Regen in der widerstehenden Luft sehr rasch eine konstante Geschwindigkeit an, die wir in erster Annäherung proportional der elektrischen Feldstärke e setzen. Das sogenannte Ohmsche Gesetz

$$
\mathfrak{i}=\lambda_{\mathrm{e}}
$$

ist daher nichts anderes als eine besonders einfache stationäre Lösung der Gleichungen der Mechanik, bei zu Grunde legen der dynamischen Gleichung 4).

Damit ist der geschlossene Gesetzeszusammenhang nachgewiesen, den die Maxwellsche Theorie und Mechanik bilden, und wir können jetzt im Abschnitt 2 daran gehen, unter Benutzung der aufgestellten Gleichungen. von den bekannten Maßeinheiten der Mechanik zu denen der Elektrodynamik vorzudringen.

2. Elektrostatische und elektromagnetische Maßsysteme. Es seien durch Aufweisen von ganz individuellen Gegenständen, Naturvorgängen usw. in immer wieder reproduzierbarer Weise die Maßeinheiten von Raum, Zeit und Kraft festgelegt, und zwar in der Folge für den Raum das $\mathrm{cm}$ durch das Urmeter in Paris, für die Zeit die sec durch die Umlaufszeit der Erde um die Sonne und für die Kraft die Dyne als Kraft, die der Masse von $\mathrm{I} \mathrm{cm}^{3}$ der Substanz reines Wasser bei der und der Temperatur usw. die Beschleunigung $\mathrm{I} \mathrm{cm} / \mathrm{sec}^{2}$ erteilt. Derartige ganz grobe Empirie scheint die notwendige Wurzel aller Maßeinheiten zu sein; die ihr anhaftende Willkür die eine — nicht die einzige Quelle der Möglichkeit verschiedener Maßsysteme.

Die dynamische Gleichung 4) bestimmt zweierlei: das eine ist die Kraft $\mathrm{k}=\varrho \mathrm{e}$ auf eine elektrische Ruhdichte in einem elektrischen Felde; das zweite die Kraft $\mathrm{k}=[\mathfrak{\mathfrak { t }}, \mathfrak{b}]$, die ein Magnetfeld auf eine Leitungsstromdichte ausübt. Beide Tatsachen stehen sich dual gegenüber, haben aber a priori rein gar nichts miteinander zu tun. 
Sie sind vom Standpunkt der bis jetzt entwickelten Theorie als zwei ganz verschiedene Phänomene anzusprechen, und jedes ist genau gleich berechtigt, uns Ausgangspunkt zu sein auf dem Wege, der aus dem Gebiet der mechanischen Größen hinüberführt in die Elektromagnetik.

Maßsysteme, die ausgehen von $\mathrm{k}=\varrho \mathrm{e}$, heißen in der Geschichte der Physik elektrostatische im Gegensatz zu den elektromagnetischen, die an $k=[\mathfrak{i}, \mathfrak{b}]$ anknüpfen.

a) Die elektrostatischen Maßsysteme.

Ausgehend von der Gleichung $\mathrm{k}=\varrho$ e führen uns die Feldgesetze I) und die Materialgleichungen 3) auf einem ganz bestimmten Wege durch das ganze System der Größen $\mathfrak{e}, \mathfrak{h}, \mathfrak{b}, \mathfrak{b}, \mathfrak{i}, \varrho$ hindurch und definieren dabei Einheit jeder folgenden nach Dimension und Größe durch die vorhergehende. Das soll jetzt gezeigt werden.

In $\mathrm{k}=\varrho \mathrm{e}$ sind $\varrho$ und $\mathfrak{e}$ vorläufig noch beide unbekannt, will sagen, noch keine von beiden ist an die empirisch gegebenen, mechanischen Einheiten Raum, Zeit und Kraft angeschlossen. Um eine Beziehung zwischen $\mathrm{k}$ und $\varrho$ allein zu erhalten, berechnen wir $e$ durch Integration der Feldgesetze aus der Ladungsverteilung $\varrho$. Wir beschränken uns dabei auf den statischen Fall. In ihm ist wegen rot $\mathrm{e}=0$ e von einem skalaren Potential $\varphi$ ableitbar

Die Substitution dieses Wertes in

$$
\mathrm{e}=-\operatorname{grad} \varphi
$$

$$
\operatorname{div} \mathfrak{b}=\varepsilon \operatorname{div} \mathfrak{e}=\varrho
$$

gibt für $\varphi$ die bekannte Potentialgleichung $-\varepsilon \Delta \varphi=\varrho$ mit der Lösung

$$
4 \pi \varepsilon \varphi=\int \frac{\varrho \mathrm{d} \tau}{\mathrm{r}} \text {. . . . . . . . . . . . . 5) }
$$

( $\mathrm{d} \tau=$ Volumenelement; $\mathrm{r}=$ absoluter Betrag des Radiusvektors vom Aufpunkt aus). Mit diesem Ausdruck von $\varphi$ wird

$$
\mathfrak{e}=\int \frac{\mathrm{r} \varrho \mathrm{d} \tau}{4 \pi \varepsilon \mathrm{r}^{3}} \quad \text { und } \quad \mathrm{k}=\varrho \int \frac{\varrho \mathrm{r}}{4 \pi \varepsilon \mathrm{r}^{3}} \mathrm{~d} \tau
$$

Diese Beziehung zwischen $\varrho$ und $\mathrm{k}$ allein - sie ist natürlich nichts anderes als eine Verallgemeinerung des Coulombschen Gesetzes der Fernwirkungstheorie - gestattet, die elektrostatische Einheit der Ladungsdichte $\varrho$ durch die mechanischen Größen zu definieren.

Alle in diesem $\mathrm{MaB}$ angegebenen Größen versehen wir in Zukunft mit dem Index e.

Aus $\varrho_{\mathrm{e}}$ folgt vermöge $\mathrm{k}=\varrho_{\mathrm{e}} \mathfrak{e}_{\mathrm{e}}$ die Feldstärke $\mathrm{e}_{\mathrm{e}}$, aus $\delta_{\mathrm{e}}=\varepsilon e_{\mathrm{e}}$ die Verschiebung $\delta_{\mathrm{e}}$. Das Feldgesetz $I$ a) vermittelt aus $\mathfrak{b}_{e}$ die Größen $\mathfrak{h}_{e}$ und $\mathfrak{i}_{\theta}$ und kraft des Induktionsgesetzes Ic) folgt aus $e_{\boldsymbol{e}}$ endlich die letzte Größe $\mathfrak{b}_{\mathrm{e}}$. Die Bestimmung von $\mathfrak{b}_{\mathrm{e}}$ aus $\varrho_{\mathbf{e}}$ direkt vermöge $\mathrm{Ib}$ ) fuihrt, da wir $\mathrm{Ib}$ ) zur Ermittlung des Integrales 5) schon verwendet haben, zum selben Resultat.

Auf die angedeutete Weise lassen sich natürlich eine ganze Menge elektrostatischer Maßsysteme definieren, je nach der Wahl der willkürlichen Konstanten, die wir uns immer beim Übergang von einer Größe zur folgenden in der den Übergang vermittelnden, unabhängigen Gleichung eingeschaltet zu denken haben.

Beispielsweise die Konstante $\varepsilon$ dürfen wir für eine Eichsubstanz nach Größe und Dimension willkürlich festsetzen. Im sogenannten absoluten elektrostatischen Maßsystem, wie es von $\mathrm{Gau} B$ begründet wurde, wählte man die Dielektrizitätskonstante $\boldsymbol{\varepsilon}$ als reine Zahl und zwar derart, $\mathrm{daB}$ im leeren Raum $\varepsilon=\mathrm{I}$ also $\mathfrak{\delta} \equiv \mathrm{e}$ wird. Auch über die Konstanten in den Maxwellschen Gleichungen — wir haben sie der Bequemlichkeit wegen alle gleich eins geschrieben, haben uns aber stets daran zu erinnern, daß sie noch unserer Willkür unterworfen sind - wird verfügt, und zwar so: 


$$
\begin{aligned}
& \left.\operatorname{roth}-\frac{\partial \mathrm{b}}{\partial \mathrm{t}}=4 \pi \mathrm{i} \quad . \quad . \quad . \quad . \quad . \quad . \quad . \quad .6 \mathrm{6}\right) \\
& \operatorname{div} b=4 \pi \varrho . . .6 . . .6 \mathrm{~b}) \\
& \left.\operatorname{rot} e+\frac{\partial \mathfrak{b}}{\partial t}=0 \quad \ldots . . . . . .66 \mathrm{c}\right) \\
& \operatorname{div} \mathfrak{b}=0 \quad \text {. . . . . . . . . . 6d) }
\end{aligned}
$$

Der Falstor $4 \pi$ bringt den entsprechenden in den Potentialausdrücken zum Verschwinden. $\mathrm{Zu} \mathrm{5)}$ existiert eine analoge Formel für das Vektorpotential eines Strom: systems. Potentiale und Coulombsches Gesetz erscheinen so in möglichst einfacher Gestalt. Das war die Absicht und war zur Zeit von Gauß und auch später so lange noch gerechtfertigt, als man den Fernwirkungsgesetzen fundamentale Bedeutung zuschrieb. Heute sind diese erkannt als ganz spezielle Integrale der viel allgemeinern Feldgleichungen, und es ist für Theoretiker wie Praktiker, die beide ausschließlich mit den letzteren operieren, höchst unbequem den Faktor $4 \pi$ in Ausdrücken mitschleppen zu müssen, wo er nicht hingehört. Doch davon später.

Es gelang uns, sämtliche Größen $\mathfrak{i}, \varrho, \mathfrak{e}, \mathfrak{h}, \mathfrak{d}, \mathfrak{b}$ im absoluten, elektrostatischen Maßsystem zu definieren, ohne daß wir die zweite Materialgleichung $\mathfrak{b}=\mu \mathfrak{h}$ noch die andere Hälfte der dynamischen Gleichung $k=[\mathfrak{i}, \mathfrak{b}]$ benutzt hätten. Es wäre sehr sonderbar, wenn die Gleichungen in der einfachen Gestalt, in der wir sie hingeschrieben, wirklich zu Recht bestünden. Es ist wohl wahrscheinlicher, daß in ihnen noch gewisse Maßsystemskonstanten auftreten werden, die wir vorläufig noch nicht kennen. Um sie zu ermitteln, sind wir gezwungen, zuerst

zu entwickeln.

\section{b) Das elektromagnetische Maßsystem}

Wir beschränken uns diesmal sogleich auf das absolute, elektromagnetische Maßsystem von GauB, in welchem die Permeabilität $\mu$ eine reine Zahl im leeren Raume zu eins wird, und in welchem über die Konstanten in den Feldgesetzen in derselben Weise verfügt wird wie in a). Die in ihm definierten Größen versehen wir mit dem Index $\mathrm{m}$.

Wir gehen aus von der Gleichung $\mathrm{k}=\left[\mathfrak{i}_{\mathrm{m}}, \mathfrak{b}_{\mathrm{m}}\right]$ und drücken durch Integration der Feldgesetze im Fall einer stationären Stromverteilung die Induktion $\mathfrak{b}_{\mathbf{m}}$ aus durch die räumliche Verteilung von $\mathfrak{i}$.

Die Gleichung

mit der Nebenbedingung

$$
\operatorname{rot} \mathfrak{h}=\frac{\mathrm{I}}{\mu} \operatorname{rot} \mathfrak{b}=4 \pi \mathfrak{i}
$$

geht durch den Ansatz

$$
\operatorname{div} \mathfrak{b}=0
$$

über in die Potentialgleichung

$$
\mathfrak{b}=\operatorname{rot} \mathfrak{f}
$$$$
-\frac{\mathrm{I}}{\mu} \Delta \mathfrak{i}=4 \pi \mathfrak{i}
$$

mit dem Vektorpotential

$$
\mathrm{f}=\mu \int \frac{\mathfrak{i} \mathrm{d} \tau}{\mathrm{r}}
$$

als Integral $[\mathrm{d} \tau$ und $\mathrm{r}$ dieselbe Bedeutung wie in 5)]. Damit wird

$$
\mathfrak{b}_{\mathrm{m}}=\mu \int \frac{\left[\mathrm{i}_{\mathrm{m}}, \mathfrak{r}\right]}{\mathrm{r}^{3}} \mathrm{~d} \tau
$$

und seine Substitution in die dynamische Gleichung gibt die erwünschte Beziehung zwischen $\mathrm{k}$ und $\mathrm{i}_{\mathrm{m}}$ allein — im wesentlichen eine Verallgemeinerung des Biot-Savartschen Gesetzes. Sie legt die elektromagnetische Maßeinheit der Stromdichte $\dot{t}_{m}$ 
fest. Aus $k=\left[\mathfrak{i}_{m}, \mathfrak{b}_{m}\right]$ folgt $\mathfrak{b}_{m}$, dann über die Materialgleichung $\mathfrak{h}_{m}$, aus $\mathfrak{h}_{m}$ vermöge Ia) $\mathfrak{b}_{\mathfrak{m}}$, aus $\mathfrak{b}_{\mathfrak{m}}$ nach $I$ b) $\varrho_{m}$ und schließlich aus dem Induktionsgesetz auch $e_{m}$. Die Bestimmung von $\mathfrak{h}_{\mathrm{m}}$ vermittelst $\mathrm{I}$ a) aus $i_{\mathrm{m}}$ direkt, ergibt dasselbe Resultat, da ja der obige Ausdruck für $\mathfrak{b}_{\mathrm{m}}$ als Integral der Gl. Ia) gewonnen wurde.

Wieder sind alle Größen definiert und wieder haben wir zwei Gesetze nicht benutzt, und zwar diesmal $\mathfrak{b}=\varepsilon \mathrm{e}$ und $\mathrm{k}=\varrho \mathrm{e}$. Es erhebt sich auch hier die Frage, mit was für Konstanten sie zu versehen sind, damit sie auch im elektromagnetischen Maßsystem gültig bleiben.

c) Der Zusammenhang zwischen elektrostatischen und elektromagnetischen Maßsystemen.

Führen wir die unter a) und b) angegebenen Rechnungen wirklich durch, so finden wir, $d a B$ die Dimensionen von $\varrho_{e}$ und $\varrho_{m}$ bzw. $i_{e}$ und $\mathfrak{i}_{m}$ sich je unterscheiden um eine Geschwindigkeit. Setzen wir etwa

$$
\frac{\varrho_{\mathrm{e}}}{\varrho_{\mathrm{m}}}=\mathrm{c}_{1} \frac{\mathrm{cm}}{\mathrm{sec}} \quad \frac{\dot{\mathrm{i}}_{\mathrm{e}}}{\dot{\mathrm{i}}_{\mathrm{m}}}=\mathrm{c}_{2} \frac{\mathrm{cm}}{\mathrm{sec}}
$$

Unabhängig vom Maßsystem faßt die moderne Elektrizitätslehre die Stromdichte $i$ auf als eine mit der Geschwindigkeit $\mathfrak{v}$ begabte Ladungsdichte $\varrho$

$$
\begin{aligned}
i_{\mathrm{e}} & =\varrho_{\mathrm{e}} \mathfrak{v} \\
\mathfrak{i}_{\mathrm{m}} & =\varrho_{\mathrm{m}} \mathfrak{v} .
\end{aligned}
$$

Das hat zur Folge, daß

$$
\frac{\varrho_{\mathrm{e}}}{\varrho_{\mathrm{m}}}=\frac{\dot{\mathrm{i}}_{\mathrm{e}}}{\mathrm{i}_{\mathrm{m}}}=\mathrm{c}_{1}=\dot{\mathrm{c}}_{\mathrm{2}}=\mathrm{c} \text {. }
$$

Der direkte Versuch: man mißt ein und dieselbe Elektrizitätsmenge das eine Mal elektrostatisch durch die Kraft, die sie ruhend in einem elektrischen Felde erfährt, das andere Mal elektromagnetisch durch die Kraft, die beim Durchfliegen eines Magnetfeldes dieses auf sie ausübt, ergibt $c==3 \cdot 10^{10} \mathrm{~cm} / \mathrm{sec}=$ der Lichtgeschwindigkeit im leeren Raum; ein Resultat, das, wie wir sehen werden, auch direkt der Theorie zu entnehmen ist.

Jede der Zustandsgrößen ist im elektrostatischen bzw. elektromagnetischen Maßsystem angeschlossen an $\varrho_{\mathrm{e}}$ bzw. $\dot{i}_{\mathrm{m}}$. Wir sind also imstande aus obigen Beziehungen für Strom- und Ladungsdichte das Verhältnis des Wertes im einen zum Werte im andern System für alle übrigen Größen sofort anzugeben.

$$
\frac{\varrho_{\mathrm{e}}}{\varrho_{\mathrm{m}}}=\frac{\dot{t}_{\mathrm{e}}}{\mathfrak{i}_{\mathrm{m}}}=\frac{\mathfrak{b}_{\mathrm{e}}}{\mathfrak{b}_{\mathrm{m}}}=\frac{\mathfrak{h}_{\mathrm{e}}}{\mathfrak{h}_{\mathrm{m}}}=\frac{\mathfrak{b}_{\mathrm{m}}}{\mathfrak{b}_{\mathrm{e}}}=\frac{\mathfrak{e}_{\mathrm{m}}}{\mathfrak{e}_{\mathrm{e}}}=\mathrm{c} .
$$

Jetzt ist es möglich, die Materialgleichungen und die dynamische Gleichung, soweit wir sie in einem System noch nicht kennen, aus den entsprechenden des andern herzuleiten. Denn es gilt ja

und folgt daraus

$$
\mathfrak{b}_{\mathrm{e}}=\varepsilon \mathfrak{e}_{\mathrm{e}} \quad \mathfrak{b}_{\mathrm{m}}=\mu \mathfrak{h}_{\mathrm{m}}
$$

Ebenso aus

$$
\begin{array}{rlrl}
\mathfrak{b}_{\mathrm{m}}=\frac{\varepsilon}{\mathrm{c}^{2}} \mathrm{e}_{\mathrm{m}} & \mathfrak{b}_{\mathrm{e}}=\frac{\mu}{\mathrm{c}^{2}} \mathfrak{h}_{\mathrm{e}} . \\
\mathrm{k}=\varrho_{\mathrm{e}} \mathrm{e}_{\mathrm{e}} & \text { und } & \mathrm{k}=\left[\mathfrak{i}_{\mathrm{m}}, \mathfrak{b}_{\mathrm{m}}\right] \\
\mathrm{k}=\varrho_{\mathrm{m}} \mathrm{e}_{\mathrm{m}} & \mathrm{k}=\left[\mathfrak{t}_{\mathrm{e}}, \mathfrak{b}_{\mathrm{e}}\right] .
\end{array}
$$

Damit ist die Gesamtheit der Größen und Gleichungen im einen wie im andern System ermittelt und auch der Zusammenhang zwischen beiden hergestellt.

Es ist bekannt, daß die Maxwellsche Theorie das wellenartige Fortschreiten elektromagnetischer Zustände im leeren Raum voraussieht, ein Vorgang, den man in 
der Wirklichkeit mit großem Erfolge als Lichtausbreitung deutete. Bei seiner rechnerischen Verfolgung im einen oder andern Maßsystem werden die Materialgleichungen benützt, und gerade die Konstante $c$ spielt dabei die Rolle der Fortpflanzungsgeschwindigkeit des Zustandes. In diesem Verstande verlangt die Theorie selbst, daß c die Lichtgeschwindigkeit sei.

3. Die Maßsysteme der theoretischen Physik. Dem Theoretiker liegt weniger an Bequemlichkeit im praktischen Rechnen als vielmehr an der möglichst adäquaten mathematischen Darstellung der Vorgänge in der Natur. So ist ihm das Maßsystem am liebsten, das keine willkürlichen Konstanten mehr enthält, oder falls das nicht erreichbar ist, so sollen sie im Formelsystem wenigstens an ihrer naturgemäßen Stelle auftreten. Was damit gemeint ist, werden die folgenden Überlegungen zeigen.

Z. B. den Faktor $4 \pi$ wird er aus den Feldgesetzen in die Potentialausdrücke abschieben. Man möchte verlangen, daß er auch in diesen verschwinde. Schon Heaviside zeigte, was da geschehen müßte. Im Grunde rührt er daher, daß die Kugel vom Radius I die Oberffäche $4 \pi$ hat. Es steht mir natürlich frei, als Flächeneinheit die Oberfläche der Kugel vom Radius I zu wählen, statt wie bisher den Inhalt des Quadrates der Kante I. Gegenüber den Unbequemlichkeiten eines solchen Flächenmaßes wird auch der Theoretiker sich gerne mit dem Faktor $4 \pi$ in den Potentialausdrücken abfinden.

Eine weitere Willkür der Gaußschen Maßsysteme steckt im Faktor c, der Lichtgeschwindigkeit. Man hört hin und wieder: Wie kommt es, daß die Lichtgeschwindigkeit gerade $3, \mathrm{IO}^{10} \mathrm{~cm} / \mathrm{sec}$ beträgt? Offenbar ist die Frage selbst verkehrt gestellt, denn in Wahrheit ist es doch so:

Es gibt eine ausgezeichnete Geschwindigkeit in der Welt, das ist die Ausbreitungsgeschwindigkeit des Lichtes im leeren Raum. Sie ist unabhängig von der Richtung, in der ich einen Lichtstrahl loslasse; ja noch mehr, sie ergibt sich sogar unabhängig von der Bewegung der Erde; denn Michelson und Morley haben mit ạßerordentlicher Genauigkeit zwischen den Geschwindigkeiten in Richtung der Erdbahntangente einerseits und senkrecht dazu andererseits keine Differenz feststellen können. Dieser Sachverhalt fand seinen mathematischen Ausdruck in der speziellen Relativitätstheorie. Damit ist aber die Möglichkeit gegeben, die Maßeinheiten von Raum und Zeit aufeinander zurückzuführen. Z. B. ich halte fest am LängenmaB $I \mathrm{~cm}$. Dann ist die naturgemäße Einheit der Zeit, diejenige, die verstreicht, während das Licht die Strecke von I cm durchläuft; oder umgekehrt, ich halte fest an der sec und bekomme als Raummaß die Strecke, die das Licht in I sec zurücklegt. Wir sehen, im ersten Fall haben Raum- und Zeitstrecken dieselbe Dimension [cm], im zweiten Fall dieselbe Dimension [sec], und für beide Fälle wird jede Geschwindigkeit eine reine Zahl, nämlich ihr Verhältnis zu der per Definition gleich I gesetzten Lichtgeschwindigkeit. In diesem natürlichen Maßsystem fallen die elektrostatischen und elektromagnetischen untrennbar zusammen. In ihm rechnet der Theoretiker von heute. Die Faktoren $\frac{I}{c^{2}}$ in den Materialgleichungen werden zu I und ein Blick auf die Feldgesetze und die dynamischen Gleichungen zeigt, daß $\mathfrak{i}$ und $\varrho$ dieselbe Dimension [Kraftdichte ${ }^{\frac{1}{2}}$ Strecke $e^{-\frac{1}{2}}$ ] und ebenso die 4 Vektoren $\mathfrak{e}, \mathfrak{h}, \mathfrak{b}, \mathfrak{b}$ auch ein und dieselbe Dimension [Kraftdichte ${ }^{\frac{1}{2}}$ Strecke $e^{\frac{1}{2}}$ ] haben. "Strecke" bedeutet hier sowohl Raum- wie Zeitstrecke. Alle willkürlichen Konstanten in den fundamentalen Beziehungen sind verschwunden.

Bevor der Mensch merkte, daß die Natur ihm ermöglicht auf diese absolute Weise Raum- und Zeitstrecken miteinander zu verknüpfen, definierte er willkürlich als Längeneinheit I cm, als Zeiteinheit I sec, und wählte damit an Stelle der natürlichen Geschwindigkeitseinheit, der Lichtgeschwindigkeit bis auf einen irrelevanten Proportionalitätsfaktor die Bahngeschwindigkeit der Erde als Maß. Die Frage, von der wir ausgingen, lautet also richtig gestellt so: Wie kommt es, daß die Bahngeschwindig- 
keit der Erde bezogen auf die Lichtgeschwindigkeit gerade den Wert hat, den wir messen? Thre Beantwortung ist nicht Sache der Physik, gehört vielmehr in die Erdgeschichte, in die Geschichte unseres Planetensystems.

Um dem hier Dargestellten auch den letzten metaphysischen Schimmer zu nehmen, sei an einem geläufigeren Beispiele gezeigt, wie die Physik schon oft an andern Begriffen genau denselben Schritt vollzogen hat. Es gab eine Zeit, da galt die Wärme als ein besonderer Stoff, und ihre Einheit wurde definiert als die Menge Fluidum, die einem Gramm Wasser zuzuführen ist, um seine Temperatur von $\mathrm{o}^{0}$ auf $\mathrm{I}^{0}$ zu erwärmen. Mit der Entdeckung des Energiesatzes und mit der Schaffung der kinetischen Theorie der Materie, die die Wärme betrachtet als kinetische Energie sehr kleiner Massen (Moleküle), war der Weg offen, aus den mechanischen Einheiten die Maßein. heiten der Wärme zu definieren. Der Übergang vom alten Fluidummaßsystem zum mechanischen wird vermittelt durch das mechanische Wärmeäquivalent A Erg/cal, dessen Größe natürlich keine universelle Bedeutung hat, sondern nur zusammenhängt mit der besonderen Konstitution des Wassers. Genau im selben Sinne kann man sagen, die Lichtgeschwindigkeit c sei das Äquivalent von Raum- und Zeitstrecken.

Die Geschwindigkeiten, die uns auf Erden zur Verfügung stehen, sind mit wenigen Ausnahmen verschwindend klein relativ zur Lichtgeschwindigkeit, bzw. die Zeitstrecken, die wir zu messen haben, außerordentlich groß gegenüber den Zeitstrecken, die den praktisch vorkommenden Raumstrecken äquivalent sind. Es ist daher oft auch dem Theoretiker angenehm, in $\mathrm{cm}$ und $\mathrm{sec}$ zu rechnen, nie aber würde er sich dazu verstehen, die dadurch auftretende Konstante $\mathrm{c}$ in die Materialgesetze zu komprimieren, wie wir es getan haben. Der Übergang von dem ganz rationalen Maßsystem mit $\mathrm{c}=\mathrm{I}$ zum System $\mathrm{c}=3 \cdot 10^{10} \mathrm{~cm} / \mathrm{sec}$ erscheint ihm vielmehr als eine Transformation der Zeiteinheit (falls wir das Längenmaß $\mathrm{I} \mathrm{cm}$ vorgeben). Bedeutet nämlich $\mathrm{t}_{\mathbf{1}}$ die Lichtzeit gemessen in $\mathrm{cm}, \mathrm{t}$ aber die sec-Zeit, so ist offenbar $t_{1}=\mathrm{ct}$. Führen wir in den Feldgesetzen und der dynamischen Gleichung des natürlichen Maßsystems, überall da wo die Zeit auftritt, diese Substitution durch, so erhalten wir als Gleichungen eines in der theoretischen Physik ebenfalls sehr gebräuchlichen Maßsystems

$$
\begin{gathered}
\operatorname{rot} \mathfrak{h}-\frac{\mathrm{x}}{\mathrm{c}} \frac{\partial \mathfrak{b}}{\partial \mathfrak{t}}=\varrho \frac{\mathfrak{b}}{\mathrm{c}}=\frac{\mathfrak{i}}{\mathrm{c}} \\
\frac{\operatorname{div} \mathfrak{b}=\varrho}{\operatorname{rot} \mathfrak{e}+\frac{\mathrm{I}}{\mathrm{c}} \frac{\partial \mathfrak{b}}{\partial \mathrm{t}}=0} \\
\operatorname{div} \mathfrak{b}=0 \\
\mathfrak{b}=\varepsilon \mathfrak{b} \quad \mathfrak{b}=\mu \mathfrak{h} \\
\mathrm{k}=\varrho \mathfrak{e}+\left[\frac{\mathfrak{i}}{\mathrm{c}}, \mathfrak{b}\right]
\end{gathered}
$$

Die Veltoren $\mathfrak{e}, \mathfrak{h}, \mathfrak{b}, \mathfrak{b}$ haben nach wie vor die Dimension [Kraftdichte ${ }^{\frac{1}{2}}$ Strecke $\frac{1}{2}$ ]. Auch die Dimension von $[\varrho]=\left[\right.$ Kraftdichte $^{\frac{1}{2}}$ Strecke $\left.{ }^{-\frac{1}{2}}\right]$ ist erhalten geblieben, stimmt aber diesmal überein mit der Dimension von $\frac{\dot{t}}{c}$ entsprechend dem Umstand, daß in $\mathfrak{t}=\varrho \mathfrak{b}$ die Geschwindigkeit $\mathfrak{v}$ jetzt keine reine $Z$ ahl mehr bedeutet.

Auch dieses Maßsystem vereinigt die elektrostatischen und elektromagnetischen in sich,

4. Das Maßsystem der Elektrotechnik von Heaviside und Cohn. Für die Bedürfnisse der Praxis kommen bei der Wahl eines Maßsystems ganz andere Gesichtspunkte in Betracht als die, die wir im vorhergehenden Paragraphen verwirklicht haben. 
Der Praktiker bedarf ursprünglich Einheiten, die von derselben Größenordnung sind wie die zu messenden Werte. Sie sollen außerdem im Laboratorium leicht und mit großer Genauigkeit reproduzierbar sein. Heute aber ist er vor allem durch eine in unzähligen Vorschriften, Gesetzen, Normalien und Meßinstrumenten zur Unabänderlichkeit erstarrte Konvention gebunden, die fast allein den engen Raum begrenzt, in dem es ihm trotzdem gelang sein Maßsystem zur Not den Verhältnissen der Gegenwart anzupassen. Von dieser Konvention haben wir jetzt zu reden.

Die meisten elektrotechnischen MeBinstrumente beruhen auf der dynamischen Wirkung elektrischer Ströme. Das elektrotechnische Maßsystem ist daher mit Recht ein elelktromagnetisches. Es ist sogar genau dasselbe, wie das in Abschnitt $2 \mathrm{~b}$ entwickelte; nur treten in ihm an Stelle der praktisch zu kleinen Spannungseinheit das Io ${ }^{8}$ mal größere Volt, an Stelle der Stromeinheit das Io mal kleinere Ampere. Die letztere Verschiebung ist wirklich nur historisch zu begreifen und hängt damit zusammen, daß man von der seinerzeit festgelegten Widerstandseinheit eines Quecksilberfadens von I $m$ Länge und $\mathrm{Imm}^{2}$ Querschnitt nicht allzusehr abweichen wollte.

Jetzt notieren wir die Gleichungen des elektromagnetischen Systems noch einmal, präparieren sie aber schon auf die Einführung von Volt $/ \mathrm{cm}$ und Amp. $/ \mathrm{cm}^{2}$ als Einheiten von e bzw. $i$

$$
\begin{aligned}
& \operatorname{rot} \mathfrak{h}_{\mathrm{m}}-\frac{\partial \delta_{\mathrm{m}}}{\partial \mathrm{t}}=\frac{4 \pi}{10}\left(\text { IO }_{\mathrm{m}}\right) \\
& \operatorname{div} \delta_{m}=\frac{4 \pi}{I 0}\left(\text { Io } \varrho_{m}\right) \\
& I 0^{8} \operatorname{rot}\left(\mathfrak{e}_{\mathrm{m}} I 0^{-8}\right)-\frac{\delta \mathfrak{b}_{\mathrm{m}}}{\partial \mathrm{t}}=0 \\
& \operatorname{div} \mathfrak{b}_{\mathrm{m}}=0 \\
& \mathfrak{b}_{\mathrm{m}}=\frac{\varepsilon}{\mathrm{C}^{2}} \mathrm{IO}^{8}\left(\mathrm{e}_{\mathrm{m}} \mathrm{IO}^{-8}\right) \quad \mathfrak{b}_{\mathrm{m}}=\mu \mathfrak{h}_{\mathrm{m}} \\
& \mathrm{k}=\mathrm{IO}^{7}\left(\mathrm{IO} \varrho_{\mathrm{m}}\right) \cdot\left(\mathrm{e}_{\mathrm{m}} \mathrm{IO}^{-8}\right)+\frac{\mathrm{I}}{\mathrm{IO}}\left[\mathrm{IOI}_{\mathrm{m}}, \mathfrak{b}_{\mathrm{m}}\right]
\end{aligned}
$$

Tragen die Größen im technischen Maß gemessen den Index $t$, so ist

Verlangen wir, daß

$$
\begin{aligned}
\text { Io } \mathfrak{i}_{\mathrm{m}} & =\mathfrak{i}_{\mathrm{t}} \text { in Amp. } / \mathrm{cm}^{2} \\
\text { IO }^{-8} \mathrm{e}_{\mathrm{m}} & =\mathrm{e}_{\mathrm{t}} \text {,Volt } / \mathrm{cm} .
\end{aligned}
$$

so folgt

Definieren wir endlich

$$
\begin{aligned}
& \dot{t}_{\mathrm{t}}=\varrho_{\mathrm{t}} \mathfrak{v} \\
& \text { Io } \varrho_{\mathrm{m}}=\varrho_{\mathrm{t}} \text { in Coulomb } / \mathrm{cm}^{3} .
\end{aligned}
$$

$$
\begin{aligned}
& \text { Io } \mathfrak{h}_{\mathrm{m}}=\mathfrak{h}_{\mathrm{t}} \text { in Amp. } / \mathrm{cm} \\
& \frac{\mathrm{Io}}{4 \pi} \mathfrak{b}_{\mathrm{m}}=\mathfrak{b}_{\mathrm{t}}, \text { Coulomb } / \mathrm{cm}^{2} \\
& \mathrm{IO}^{-8} \mathfrak{b}_{\mathrm{m}}=\mathfrak{b}_{\mathrm{t}}, \quad\left[\text { Voltsec } / \mathrm{cm}^{2}=\text { Maxwell } / \mathrm{cm}^{2}\right],
\end{aligned}
$$

so erscheinen die Gesetze in der ganz einfachen Gestalt

$$
\begin{aligned}
\operatorname{roth}_{\mathrm{t}}-\frac{\partial \mathrm{D}_{\mathrm{t}}}{\partial \mathrm{t}} & =\mathrm{i}_{\mathrm{t}} \\
\operatorname{div} \grave{D}_{\mathrm{t}} & =\varrho_{\mathrm{t}}
\end{aligned}
$$


Dabei bedeuten

$$
\begin{gathered}
\operatorname{rot}_{\mathfrak{t}}+\frac{\partial \mathfrak{b}_{\mathfrak{t}}}{\delta \mathrm{t}}=0 \\
\operatorname{div} \mathfrak{b}_{\mathfrak{t}}=0 \\
\begin{array}{l}
\mathfrak{b}_{\mathfrak{t}}=\varepsilon_{0} \varepsilon e_{\mathfrak{t}} \\
\mathfrak{b}_{\mathrm{t}}=\mu_{0} \mu \mathfrak{h}_{\mathrm{t}}
\end{array} \quad \mathrm{k}=\varrho_{\mathrm{t}} \mathfrak{e}_{\mathrm{t}}+\left[\mathfrak{t}_{\mathfrak{t}}, \mathfrak{b}_{\mathrm{t}}\right]
\end{gathered}
$$

$$
\begin{aligned}
& \varepsilon_{0}=\frac{\mathrm{IO}^{9}}{4 \pi \mathrm{c}^{2}}=0,884 \cdot \mathrm{IO}^{-13}[\text { Coulomb } / \text { Volt } / \mathrm{cm}=\mathrm{Farad} / \mathrm{cm}] \\
& \mu_{0}=\frac{4 \pi}{\mathrm{IO} 0^{9}}=\mathrm{I}, 257 \cdot \mathrm{IO}^{-8}[\mathrm{Voltsec} / \mathrm{Amp} \cdot / \mathrm{cm}=\mathrm{Henry} / \mathrm{cm}]
\end{aligned}
$$

Wir haben sie vor unseren Augen entstehen sehen und wissen, es sind bloße Maßsystemskonstanten, die wesentlich die Lichtgeschwindigkeit

$$
\mathrm{c}=\sqrt{\varepsilon_{0} \mu_{0}} \mathrm{~cm} / \mathrm{sec}
$$

enthalten. $\varepsilon$ und $\mu$ sind beides reine Zahlen und werden im leeren Raume zu I. Die Kraftdichte $\mathrm{k}$ ist nicht mehr in Dyn $/ \mathrm{cm}^{3}$, vielmehr in der $10^{7}$ mal größeren Einheit Joule $/ \mathrm{cm} / \mathrm{cm}^{3}$ gemessen.

Mit Genugtuung konstatieren wir, daß in den Feldgesetzen der Faktor $4 \pi$ verschwunden ist. Er steckt in den Materialgleichungen. . Die Konstanten $\varepsilon_{0}$ und $\mu_{0}$ sind die einzigen, die noch das Gedächtnis belasten, und auch sie braucht man eigentlich selten, da insbesondere die oft verwendete magnetische Materialgleichung meistens in Form einer Tabelle oder graphisch als Magnetisierungskurve gegeben ist. Die Einheit der Induktion $\mathfrak{b}_{\mathfrak{t}}$ ist allerdings etwas groß, so daß die praktisch vorkommenden Werte wie

$$
\mathrm{b}=\mathrm{I} 4000 \mathrm{GauB}=\mathrm{I} 4000 \cdot \mathrm{IO}^{-8}\left[\mathrm{Volt} / \mathrm{sec} / \mathrm{cm}^{2}=\mathrm{Maxwell} / \mathrm{cm}^{2}\right]
$$

sehr klein ausfallen. Doch ist kein Schaden $\mathfrak{b}$ nach wie vor in Gauß anzugeben und $\mathrm{zu}$ irgendeiner Rechnung den Wert mit der einfachen Zahl Io-8 $\mathrm{zu}$ multiplizieren.

5. Weitere in der Elektrotechnik gebräuchliche Gleichungen im praktischen Maßsystem. Es ist mir nicht darum zu tun, das, was die Überschrift angibt, in einer Tabelle zusammenzustellen. Wir werden vielmehr zuerst die differentiellen Beziehungen; die wir bis jetzt verwendet haben, ergänzen und in einem zweiten Teil zeigen, wie aus dem vollständigen Schema der Gleichungen die wichtigsten in der Elektrotechnik gebräuchlichen Beziehungen als Integrale entspringen.

Den Index $t$ lassen wir weg, da wir in diesem Abschnitt nur im praktischen Maßsystem rechnen.

Das Ohmsche Gesetz

$$
\mathrm{i}=\lambda \mathrm{e}
$$

haben wir ergänzend den Materialgleichungen beizufügen. Es gilt in dieser Gestalt, falls wir die Leitfähigkeit $\lambda$ in $1 / \mathrm{Ohm} / \mathrm{cm}$ messen.

Außer der Kraftdichte auf Strom- und Ladungsdichte erfahren Dielektrikum und Diamagnetikum noch besondere Kräfte. Der vollständige Ausdruck der Kraftdichte lautet

$$
\begin{aligned}
\mathrm{k}=\varrho \mathfrak{e}+[\mathfrak{t}, \mathfrak{b}] & -\int \operatorname{grad}\left(\varepsilon_{0} \varepsilon\right) \cdot \mathfrak{e d} \mathfrak{e} \\
& -\int \operatorname{grad}\left(\frac{-\mathrm{I}}{\mu_{0} \mu}\right) \mathfrak{b d} \mathfrak{b} .
\end{aligned}
$$

Dielektrizitätskonstante $\varepsilon$ und Permeabilität $\mu$ sind als eindeutige Funktionen des Feldes gedacht, d. h. Ferromagnetismus ohne Hysterese ist noch zugelassen. $\operatorname{grad}\left(\varepsilon_{0} \varepsilon\right)$ 
und grad $\left(\frac{-I}{\mu_{0} \mu}\right)$ sind gemeint bei räumlich konstantem Felde. Die Integrale werden erstreckt vom unpolarisierten Zustand der Materie bis zu dem Feldwerte, für den man die Kräfte berechnen will. Ein Dielektrikum bzw. Diamagnetikum erfährt also überall da Kräfte, wo die Magnetisierungskurve $\mathfrak{b}=\mathfrak{b}(\mathfrak{h})$ bzw. $\mathfrak{d}=\mathfrak{d}(\mathfrak{e})$ sich von Ort zu Ort ändert. Insbesondere übt das Feld auf die Oberfläche eines Körpers im leeren Raum oder auf die Grenzschicht zwischen zwei verschiedenen Körpern Kräfte aus, die aus der angegebenen Volumenkraft leicht zu berechnen sind. Der Kraftausdruck gilt ganz allgemein für ruhende isotrope (nicht kristallisierte) Körper in irgendeinern beliebig veränderlichen elektromagnetischen Felde. Sie umfassen dagegen nicht die Kraftwirkungen, die bei Hysterese, bei Dispersion oder bei Abhängigkeit von $\varepsilon$ und $\mu$ vom elastischen Zustand (Elektrostriktion) auftreten

In Ergänzung der dynamischen Gleichungen haben wir noch die Energiegleichung zu notieren

$$
\mathrm{w}=-\operatorname{div} \mathfrak{z}-\frac{\partial \mathrm{u}}{\partial \mathrm{t}}
$$

in Worten: Die im Volumen I pro Zeiteinheit erzeugte Joulesche Wärme $w$ ist äquivalent der gleichzeitig in das Gebiet von außen einströmenden Energie - div $\mathfrak{s}$ plus der Abnahme der elektromagnetischen Feldenergiedichte $-\frac{\partial u}{\partial t}$. Dabei ist der Joule
sche Effekt

$$
\mathrm{w}=(\mathfrak{i}, \mathrm{e}) \text { gemessen in } \mathrm{Watt} / \mathrm{cm}^{3}
$$

der Energieströmungsvektor

und die Energiedichte

$$
\mathfrak{E}=[\mathrm{e}, \mathfrak{h}] \text { in } \mathrm{Watt} / \mathrm{cm}^{2}
$$

$$
\mathrm{u}=\int(\mathrm{e}, \mathrm{d} \mathfrak{b})+\int(\mathfrak{h}, \mathrm{d} b) \text { in Joule } / \mathrm{cm}^{3} .
$$

Der Ausdruck für die Energiedichte ist denselben einschränkenden Bedingungen unterworfen, die wir für die Kraftdichte aufgestellt haben. Auch die Integrale haben dieselbe Bedeutung wie oben.

Damit sind wir beim zweiten Teile dieses Paragraphen angelangt.

a) Die Feldgesetze.

Die Anwendung der als GauBscher und Stokescher Satz bekannten Integraltheoreme der Vektorrechnung ergibt aus I) die Integralform der Feldgesetze, wie wir sie in 2) dargestellt haben.

b) Die Materialgleichungen.

Für eine Flußröhre der dielektrischen Verschiebung $\delta$, die frei von Ladungen ist, hat die Materialgleichung $\mathfrak{b}=\varepsilon_{0} \varepsilon \mathrm{e}$ das Integral

$$
\mathrm{E}=E \Psi \text {. }
$$

Darin bedeuten $\Psi$ den Verschiebungsfluß der Röhre in Coulomb, E die elektrische Spannung in Volt zwischen zwei endlich distanten Röhrenquerschnitten $q_{1}$ und $q_{2}$ und endlich $E$ den dielektrischen Widerstand definiert durch

$$
E=\int_{q_{1}}^{\mathrm{q}_{2}} \frac{\mathrm{dl}}{\mathrm{q} \varepsilon_{0} \varepsilon} \text { in }[\text { Volt } / \text { Coulomb }=\mathrm{I} / \text { Farad }] \text {. }
$$

Genau ebenso gilt als Integral von $\mathfrak{b}=\mu_{0} \mu \mathfrak{h}$ für eine magnetische FluBröhre

$$
\mathrm{M}=M \Phi, \quad \text { wo } \quad M=\int_{\mathbf{q}_{1}}^{\mathrm{q}_{2}} \frac{\mathrm{d} \mathrm{l}}{\mathrm{q} \mu_{0} \mu} \text { in }[\text { Amp./Volt/sec }=\mathrm{I} / \text { Henry }] \text {. }
$$


Und endlich aus $i=\lambda \mathrm{e}$ für einen stationären Stromfaden die Integralform des $\mathrm{Ohm}$. schen Gesetzes

$$
\left.\mathrm{E}=R \mathrm{i}, \quad \text { wo } \quad R=\int_{\mathrm{q}_{1}}^{\mathrm{q}_{2}} \frac{\mathrm{d} \mathrm{l}}{\mathrm{q} \lambda} \text { in [Volt/Amp. }=\mathrm{Ohm}\right]
$$

Im engsten Zusammenhang mit dielektrischem und magnetischem Widerstand $E$ und $M$ stehen Kapazität $\mathrm{C}$ eines Konduktors und Selbstinduktion L einer Stromschleife.

Auf einem Konduktor sitze die wahre Ladung e, die ihm gegen die unendlich ferne Kugel das Potential $\varphi$ erteilt. Für die i-te von ihm ausgehende VerschiebungsfluBröhre gilt die Gleichung $\frac{\mathrm{I}}{E_{i}}=\frac{\Psi_{1}}{\mathrm{E}}$, durch Summation über alle Röhren, in die wir uns das Feld aufgeteilt denken, resultiert

$$
\sum_{\mathrm{i}} \frac{\mathrm{I}}{E_{\mathrm{i}}}=\mathrm{C}=\frac{\sum_{\mathrm{i}} \Psi_{\mathrm{i}}}{\varphi}=\frac{\mathrm{e}}{\varphi}
$$

wo $C$ in Farad gemessen die Kapazität bedeutet.

Analog für die Stromschleife

$$
\Sigma \frac{\mathrm{I}}{M_{\mathrm{i}}}=\mathrm{L}=\frac{\sum_{\mathrm{i}} \Phi_{\mathrm{i}}}{\mathrm{i}}=\frac{\Phi}{\mathrm{i}} .
$$

wo $\mathrm{L}$ in Henry gemessen die Selbstinduktion bedeutet.

c) Die dynamischen Gleichungen.

c) Ruhende Konduktoren im homogenen Dielektrikum.

Die Kraft auf die Ladung de beträgt

$$
\mathrm{d} \Re=\mathrm{dee} \text { in Joule/cm. }
$$

Die Fंeldstärke $e$ folgt aus

$$
\mathfrak{e}=-\operatorname{grad} \varphi
$$

wo

das skalare Potential bedeutet., zu

$$
\varphi=\frac{\mathrm{I}}{4 \pi \varepsilon_{0} \varepsilon} \int \frac{\mathrm{d} \mathrm{e}}{\mathrm{r}}
$$

$$
\mathfrak{e}=\frac{\mathrm{I}}{4 \pi \varepsilon_{0} \varepsilon} \int \frac{\mathrm{rde}}{\mathrm{r}^{3}} .
$$

Sind insbesondere nur zwei Punktladungen $e_{1}$ und $e_{2}$ vorhanden, so gilt für $\Omega$ das Coulombsche Gesetz:

$$
\mathfrak{S}=\frac{\mathrm{e}_{1} \mathrm{e}_{2}}{4 \pi \varepsilon_{0} \varepsilon \mathrm{r}^{2}} \text { in Joule } / \mathrm{cm} \text {. }
$$

Die Gesamtenergie U des erzeugten Feldes beträgt

$$
\mathrm{U}=\sum_{\mathrm{i}} \frac{\mathrm{I}}{2} \varphi_{\mathrm{i}} \mathrm{e}_{\mathrm{i}} \text { in Joule. }
$$

wo $\varphi_{\mathrm{i}}$ das Potential und $\mathrm{e}_{\mathbf{i}}$ die Gesamtladung des $\mathrm{i}$-ten Konduktors bedeuten.

$\beta$ ) Konstante lineare Ströme im homogenen Diamagnetikum.

Die Kraft auf das Stromelement idl beträgt

$$
\mathrm{d} \Re=[\mathrm{id} 1, \mathfrak{b}] \text { in Joule } / \mathrm{cm} \text {; }
$$

$\mathfrak{b}$ berechnet sich vermöge des Ansatzes

$$
\mathfrak{b}=\operatorname{rot} \mathfrak{f}
$$


aus dem Vektorpotential $\mathfrak{i}$

$$
\tilde{i}=\frac{\mu_{0} \mu}{4 \pi} \int \frac{\mathrm{id} l}{\mathrm{r}}
$$

$\mathrm{zu}$

$$
\mathfrak{b}=\frac{\mu_{0} \mu}{4 \pi} \int \frac{[\mathrm{idl}, \mathfrak{r}]}{\mathrm{r}^{3}}
$$

Das ist im wesentlichen schon das Biot-Savartsche Gesetz.

Bedeuten $i_{\mathrm{i}}$ die Stärke des i-ten Stromes, $\Phi_{\mathrm{i}}$ der von ihm umschlungene magnetische Induktionsfluß, so ist die totale magnetische Energie eines Systems von linearen Strömen gegeben durch

$$
\mathrm{U}=\sum_{\mathrm{i}} \frac{\mathrm{I}}{2} \mathrm{i}_{\mathrm{i}} \Phi_{\mathrm{i}} \text { in Joule. }
$$

Fließt in einem Stromkreis unter der Spannung E der Strom i, so wird während der Zeit $\mathrm{t}$ die Joulesche Wärme

entwickelt.

$$
\mathrm{Q}=\text { Eit in Joule }
$$

6. Feld und Materie. In den Materialgleichungen $\mathfrak{b}=\varepsilon_{0} \varepsilon \mathfrak{e}, \mathfrak{b}=\mu_{0} \mu \mathfrak{h}$ wird im leeren $\operatorname{Raum} \varepsilon=\mu=\mathrm{I}$. Das verleitete dazu, $\varepsilon_{0}$ und $\mu_{0}$, von denen wir wissen, daß sie bloße Maßsystemskonstanten sind, anzusprechen als „absolute Dielektrizitätskonstante bzw. Permeabilität des Weltäthers". Ich kann mir nicht versagen, hier eines drolligen Vergleiches von Einstein zu gedenken, der die Schiefe dieser Behauptung ins rechte Licht setzt. Es sei a die Kantenlänge eines Quadrates, $\alpha$ eine sehr große Konstante. Dann ist offenbar $\mathrm{F}=\alpha \mathrm{a}^{2}$ ein Maß der Fläche des Quadrates. Die Definition ist sogar sehr vernünftig, wenn wir es in Wirklichkeit immer mit sehr großen Strecken, ảber außerordentlich kleinen Flächen zu tun haben. Was würden wir aber sagen, wenn nun einer behauptet, $a$ sei die absolute Flächenkonstante des Weltäthers? Die Konstante $\alpha$ unterliegt eben tatsächlich unserer Willkür, und es ist nur aus Opportunitätsgründen, da $\beta$ wir sie in der Regel beim Quadrat, nicht aber beim Kreis vom Radius a eins setzen. In absoluter Weise festzulegen ist nur das Verhältnis der Flächen von Quadrat mit Kante a und Kreis mit Radius a.

Dieselbe aber auch genau dieselbe Sachlage bestand in der Elektrizitätslehre: Der leere Raum war ein Kontinuum wie irgendeine andere Substanz, und was wir zu messen vermochten, war immer nur die Dielektrizitätskonstante einer Substanz relativ zu einer Eichsubstanz. Daß die Physiker für die letztere den leeren Raum gewählt und für ihn $\varepsilon_{0} \varepsilon=\mu_{0} \mu=$ I gesetzt haben, lag daran, daß er sich von Anbeginn doch vor allen andern Substanzen etwas auszeichnete: Er zeigte keine Absorption, er war streng homogen und immer in derselben Qualität reproduzierbar, die Techniker aber wählten als Muttersubstanz eine bloß gedachte, deren Dielektrizitätskonstante und Permeabilität relativ zum Äther der Physiker die ungeheuer großen Werte $\frac{I}{\varepsilon_{0}}$ bzw. $\frac{I}{\mu_{0}}$ hatte. Die Gründe dafür sind uns bekannt: Wir wünschen Maßeinheiten, die bei einfacher Gestalt der wesentlichen Gesetze den praktisch vorkommenden Werten der Größenordnung nach entsprechen.

Heute ist der Fall ein ganz anderer, heute wissen wir, daß der leere Raum eben gerade nicht ein Kontinuum ist, wie irgendeine andere Substanz. Er ist überhaupt keine Substanz, kein Äther, vielmehr die Abwesenheit alles Stofflichen. Diese negative Aussage ist das beste Zeichen für die Verlegenheit, in die die Wissenschaft gerät, wenn man von ihr verlangt, sie solle positiv erklären, was das Vakuum denn eigentlich sei. Wir wissen es nicht! Dagegen steht experimentell fest: Das Vakuum verhält sich anders als irgendeine Substanz: Es zeigt keine Absorption; die Lichtgeschwin- 
digkeit $\mathrm{c}$ in ihm ist größer als irgendeine andere uns bekannte Geschwindigkeit; sie ergibt sich beispielsweise für einen von der Sonne kommenden Lichtstrahl unabhängig davon, ob ich mich mit großer Geschwindigkeit dem Strahl entgegen- oder mit eben dieser. Geschwindigkeit von ihm weg bewege: das sonderbare Resultat des Michel. sonschen Versuches, das für einen sich bewegenden Körper - wie die einfachste Anschauung lehrt - niemals zutrifft und selbst für. Licht in andern Medien (Wasser im Versuch von Fizeau) keineswegs erfullt ist.

Die Reihe der Versuche ließe sich fortsetzen. Sie haben alle in der Relativitätstheorie ein mathematisches Kleid von so vollendeter Harmonie gefunden, daß dem Physiker von heute die vielleicht doch nicht ganz unberechtigte Hoffnung dämmert, es sei dem Geiste dereinst ein Tag beschieden, da er in der Natur sein eigen Abbild sieht.

Hätten uns zum Experimentieren nur elektrische Ladungen im leeren Raum zur Verfügung gestanden, so wären wir nie auf die Idee gekommen, die Vektoren $e$ und $b$ zu unterscheiden von den entsprechenden $\mathfrak{b}$ und $\mathfrak{h}$. Auf diesen Standpunkt haben wir uns zurückzuversetzen, denn das Vakuum ist etwas Ausgezeichnetes. Im natürlichen Maßsystem der theoretischen Physiker, das wir in der Folge wieder benutzen wollen, lauten dann die Maxwellschen Gleichungen des Vakuums

$$
\begin{aligned}
\operatorname{rot} b-\frac{\partial \mathfrak{e}}{\partial \mathrm{t}} & =\mathfrak{i}=\varrho \mathfrak{b} \\
\operatorname{div} \mathfrak{e} & =\varrho \\
\operatorname{rot} e+\frac{\partial \mathfrak{b}}{\partial \mathrm{t}} & =0 \\
\operatorname{div} \mathfrak{b} & =0 .
\end{aligned}
$$

Was ist jetzt aber die Materie? Darauf gibt uns die Elektronentheorie vorläufig folgende Antwort.

Die Materie besteht aus unzählig vielen kleinsten positiven und negativen Elektrizitätsatomen, den Elektronen, die wie Fremdkörper im Vakuum herumfliegen, ein Feld erzeugen und von diesem wieder Kräfte erfahren. Die Elektronen sind zusammengefaßt zu kleinen Planetensystemen, zu chemischen Atomen und zu Molekülen, die als ganze nach außen meist ungeladen wegen der raschen Rotationen der in ihnen enthaltenen Elektronen aber doch ein magnetisches Moment besitzen. Die Materie, sagen wir ein Stück Eisen oder Porzellan, enthält nun wieder in regelloser Anordnung, sofern wir es nicht mit einem Kristall zu tun haben, eine ungeheure Menge solcher Moleküle und zwischen ihnen, falls der Körper ein Leiter ist, noch einen ganzen Schwarm von frei beweglichen Elektronen, den Leitungselektronen. Alle zusammen erzeugen im Innern des Körpers ein ganz unübersehbar kompliziertes Feld, das sogenannte Mikrofeld, dessen Mittelwert über ein Gebiet, das viele Moleküle enthält, im unpolarisierten Zustand der Materie verschwindet.

Legen wir aber ein äußeres elektromagnetisches Feld an, so werden infolge des elektrischen Feldes die positiven Teilchen im Innern eines Moleküls in der Richtung, die negativen aber entgegen der Richtung des Feldes etwas verschobene Bahnen durchlaufen. Durch ein Flächenelement von der Größe eins senkrecht zur Feldstärke $\mathfrak{e}$ wird also durch das Polarisieren im Mittel eine bestimmte Menge elektrischer Ladungen verschoben. Wir nennen sie die elektrische Polarisation p. Sie ist ein Vektor. Die durch das beliebige Flächenelement di hindurch getretene Ladung beträgt ( $p, d \mathfrak{f}$ ).

Ein äußeres Magnetfeld übt auf die Molekularströme kleine Drehmomente aus und sucht sie zu ordnen. Die Materie wird magnetisch polarisiert. Ein Längenelement von der Größe I habe die Richtung des äußeren Magnetfeldes. Es ist klar, im unpolarisierten Zustand der Materie wird es im Mittel eine gleiche Zahl der kleinen Stromschleifen im positiven wie im negativen Sinne durchqueren, so daß die an seinen 
Enden durch diese induzierte magnetische Spannung $\int(\mathfrak{b}, \mathrm{d} l)$ verschwindet. Sobald wir aber polarisieren, werden die Moleküle etwas geordnet und die jetzt an den Enden der Länge I auftretende magnetische Spannung nennen wir den Vektor magnetische Polarisation m. An den Enden eines beliebig gerichteten Längenelementes dl induzieren die Molekularströme die magnetische Spannung ( $m, d l)$.

In die Abhängigkeit von $\mathfrak{p}$ wie $m$ vom Felde geht die ganze molekulare Konstruktion der Materie ein.

Wir sprachen bis jetzt immer von einem äußeren Felde. Darunter sind natürlich die Mittelwerte $\overline{\mathfrak{b}}$ und $\overline{\mathfrak{e}}$ des Mikrofeldes $\mathfrak{b}$ und $\mathrm{e}$, gebildet über ein Gebiet, das viele Moleküle enthält während einer Zeit, die viele Umläufe der Elektronen umspannt, zu verstehen. Mit dieser Definition ist es möglich, die Maxwellschen Gleichungen im Innern der Materie aus denen des leeren Raumes plausibel zu machen.

Wir beginnen mit der letzten der Gleichungen

$$
\operatorname{div} \mathfrak{b}=0 \text {. }
$$

Für jede unendlich kleine mathematische Oberfläche ist der Induktionsfluß null; aber auch für jede endliche und damit auch für den Mittelwert der Induktion

$$
\operatorname{div} \bar{b}=0 \text {. }
$$

Genau ebenso verhält es sich mit dem Induktionsgesetz; es gilt für unendlich kleine Integrationsschleifen, aber auch für endliche und damit schließlich für diè Mittelwerte

$$
\operatorname{rot} \overline{\mathrm{e}}+\frac{\partial \overline{\mathrm{b}}}{\partial \mathrm{t}}=0 \text {. }
$$

Die linke Seite der Gleichung

$$
\operatorname{div} e=\varrho
$$

gilt unverändert für den Mittelwert. $\varrho$ bedeutet die Elektrizitätsmenge, die im Volumen eins wirklich vorhanden ist. Sie setzt sich zusammen aus der wahren Ladungsdichte $\bar{\varphi}$ und den an die Moleküle gebundenen Ladungen, die beim Polarisieren mehr in das Volumen hinein- als herausgeströmt sind. Sie betragen nach Definition von $\mathfrak{p}$ : - divp, so daß wir erhalten:

In der letzten Gleichung endlich

$$
\operatorname{div} \overline{\mathrm{e}}=-\operatorname{div} \mathfrak{p}+\bar{\varrho}
$$

$$
\operatorname{rot} \mathfrak{b}-\frac{\partial \mathrm{e}}{\partial \mathrm{t}}=\mathfrak{i}
$$

tritt zum Leitungsstrom $\overline{\dot{i}}$ im früheren Sinne noch der Polarisationsstrom $\frac{\partial \mathfrak{p}}{\partial \mathrm{t}}$ herrührend von den Polarisationselektronen, die momentan sich verschieben und dadurch eine zusätzliche magnetische Spannung induzieren. $Z \bar{u} \overline{\mathfrak{i}}+\frac{\partial p}{\partial t}$ haben wir erst noch die magne. tische Spannung zu addieren, die durch die Molekularströme, die der Integrationsweg anschlauft, induziert wird. Sie beträgt rot $m$, so daß für die Mittelwerte folgende Gleichung besteht:

$$
\operatorname{rot} \bar{b}-\frac{\partial \overline{\mathrm{e}}}{\partial \mathrm{t}}=\operatorname{rot} \mathfrak{m}+\frac{\partial \mathfrak{p}}{\partial \mathrm{t}}+\dot{\mathrm{t}}
$$

Lassen wir jetzt die Querstriche bei den Mittelwerten der Feldgrößen wieder weg, so erhalten wir als System der Maxwellschen Gleichungen

$$
\begin{aligned}
\operatorname{rot} \mathfrak{b}-\frac{\partial \mathfrak{e}}{\partial t} & =\operatorname{rot} \mathfrak{m}+\frac{\partial \mathfrak{p}}{\partial t}+\mathfrak{i} \\
\operatorname{div} \mathfrak{e} & =-\operatorname{div} \mathfrak{p}+\varrho \\
\operatorname{rot} e+\frac{\partial \mathfrak{b}}{\partial t} & =0 \\
\operatorname{div} \mathfrak{b} & =0 .
\end{aligned}
$$


Führen wir die Substitution „dielektrische Verschiebung“ $\mathfrak{b}=\mathfrak{e}+\mathfrak{p}$, „magnetische Feldstärke" $\mathfrak{h}=\mathfrak{b}-\mathfrak{m}$ ein, so erkennen wir, daß diese Maxwellschen Gleichungen der Elektronentheorie vollkommen identisch sind mit den früheren Gleichungen I) der phänomenologischen Theorie.

Die bloß abgeleitete vermittelte Bedeutung der Vektoren $\mathfrak{b}$ und $\mathfrak{h}$ gegenüber den eigentlichen Feldgrößen $\mathfrak{e}$ und $\mathfrak{b}$ tritt jetzt klar in Erscheinung, und der ausgezeichneten Stellung des Vakuums gegenüber der Materie ist durch die Aufspaltung in $e$ und $\mathfrak{p}$, $\mathfrak{b}$ und $\mathfrak{i t}$ Rechnung getragen.

Die bisherigen Materialgleichungen finden vollen. Ersatz in den konsequenteren Beziehungen:

$$
\mathfrak{p}=\mathfrak{p}(\mathfrak{e}) \text { und } \mathfrak{n t}=\mathfrak{m}(\mathfrak{b}) \text {. }
$$

Neben der nach Abraham benannten und hier vorgetragenen Symmetrie $\{e, b\}$ $\{\delta, \mathfrak{h}\}$ der Zustandsgrößen findet man hin und wieder noch die alte Hertzsche $\{\mathfrak{e}, \mathfrak{h}\}$ $\{\mathfrak{b}, \mathfrak{b}\}$ vertreten. Die bestünde dann zu Recht, wenn die magnetische Polarisierbarkeit der Materie nicht zurückzuführen wäre auf Molekularströme, sondern auf kleine Elementarmagnete, auf Dipole von Magnetismusmengen.

Theorie und Experiment sind sich darüber einig, daß Magnetismusmengen nicht existieren und die Einführung dieser Fiktion rührt nur daher, daß das magnetische Feld permanenter Magnete zeitlich vor dem Feld elektrischer Ströme entdeckt wurde.

Dem Physiker wird an dieser Arbeit wohl nur das unter Elektrotechnikern gebräuchliche Maßsystem von Heaviside und Cohn neu sein.

Für mich beanspruche ich die Art der Darstellung und den Glauben, durch diesen Botendienst zwischen Theorie und Praxis ein Kleines beigetragen zu haben zur geistigen Befreiung des Technikers aus der Flut der unverstandenen, halb durchdrungenen Tatsachen, der er zum Verhängnis unserer Kultur auch heute noch allzuoft erliegt.

\section{Benutzte Literatur.}

E. Cohn, Das elektromagnetische Feld (Hirzel r goo).

P. Drude, Die Physik des Äthers (Enke 1912).

M. Abraham und A. Föpp1, Theorie der Elektrizität (Teubner I9Io).

H. A. Lorentz, Enzyklopädie der mathematischen Wissenschaften, V, I4.

H. Weyl, Raum, Zeit, Materie (Springer 1918). Dort auch ein Verzeiçniș der einschlägigen Literatur.

Die im Rahmen der Relativitätstheorie wohl endgültigen Ausdrücke für die elektromagnetische Polarisation und die Kraftdichte im Innern der Materie habe ich in meiner noch unveröffentlichten Promotionsarbeit gegeben.

Mit der Frage der Mafssysteme hat sich speziell Fritz Em de sehr eingehend befaft: Die

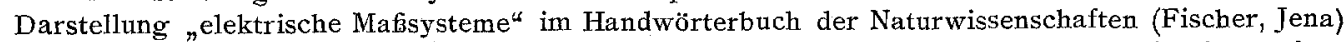
ist meines wissens seine letzte Äuferung über diesen Gegenstand. In dieser wie auch in einer seiner früheren Arbeiten ETZ I904 S. 432 findet die historische Seite der Frage, die ich gegenüber dem Prinzipiellen bewuft habe zurïcktreten lassen, eine eingehende Würdigung. Ein ziemlich vollständiges Literaturverzeichnis, auf das ich hier nur verweise, ist der letzteren Arbeit mitgegeben. 